\title{
Towards Liquid Chromatography Time-Scale Peptide Sequencing and Characterization of Post-Translational Modifications in the Negative-Ion Mode Using Electron Detachment Dissociation Tandem Mass Spectrometry
}

\author{
Frank Kjeldsen, Ole B. Hørning, Søren S. Jensen, Anders M. B. Giessing, \\ and Ole N. Jensen \\ Protein Research Group, Department of Biochemistry and Molecular Biology, University of Southern \\ Denmark, Odense, Denmark
}

\begin{abstract}
Electron@detachment@dissociation@(EDD)@of@peptide@poly-anions@is@gentle@towards@posttranslational@modifications $₫ \mathrm{PTMs}) @$ nd@roduces@redictable@nd@nterpretable@ragment@on

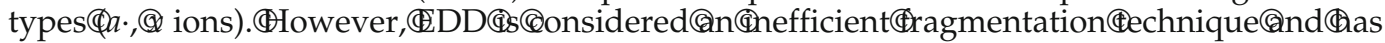
not@et(@een@mplemented@n@arge-scale@eptide@haracterization@trategies.@We@uccessfully

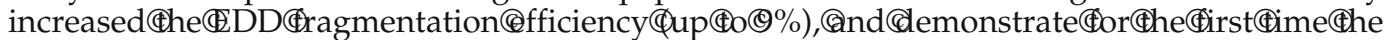
utility@of@EDD-MS/MS@in@liquid@chromatography@time-scale@experiments.@Peptides@and phosphopeptides@were@analyzed@in@both@positive-Cand@negative-ion@mode@using@electron capture/transfer@issociation $₫$ ECD/ETD)@nd@DD@n@omparison.@sing@pproximatelyđ pmol@f@্CBSA@ryptic@igest,CLC-EDD-MS/MS@equenced@44@eptides@27\%@a@equence coverage)@nd@C-ECD-MS/MS@equenced@99peptides@39\%@a@equence@overage).@Seven peptides@18\%@a@equence@overage)@rere@equenced@y@oth@DD@ndథCD.Che@elative small@overlap@of@identified@BSA@peptides@demonstrates@the@complementarity@of@the@two dissociation@modes. $\Phi$ hosphopeptide@nixtures@rom@hree@rypsin-digested@phosphoproteins

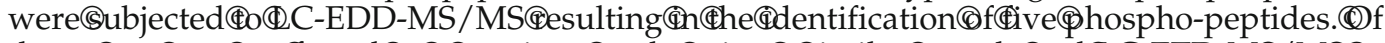
those,@ne@as@ot@ound@n@థrevious@tudy@sing@@imilar@ample@nd@C-ETD-MS/MS@n the甲ositive-ion@node.@n@his@tudy, $₫$ he®CDథragmentation@fficiency $\$ 15.7 \% @ v$.)@as@uperior

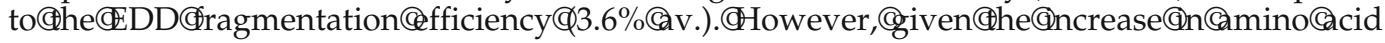
sequence@overage@nd@xtended $\oplus \mathrm{TM} @$ haracterization $₫$ he@ew@egime@f®DDథn@ombination

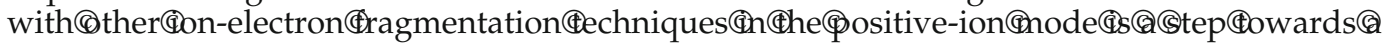
more@omprehensive@trategy@f@nalysis@n@roteome@esearch.@ (JCAm@soc@Mass@Spectrom 2008,@9,@156@1162)@(008@American@ocietyథror@Mass@sectrometry
\end{abstract}

$\mathrm{T}$ The@uman@rganism@ontains@n@stimated@3,000 protein-coding@genes@1].CThe@number@of@gene products@proteins)@s@ne@o@wo@rders@f@nag-

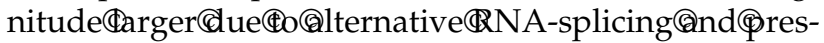
ence@f@o-@nd@ost-translational@nodifications@PTM) $[2, \mathbb{C}] . @ n @$ proteomic@tudies,@andem@mass@pectrometry (MS/MS)@s@@key@nethodologyథ4-6],@ith@lectrospray

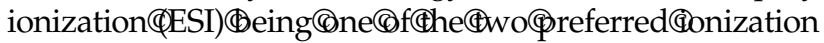
techniquesథ7-10].@n@@ypical@MS/MS@xperiment,@aseous@ations@f@proteins@r@heir@ryptic@peptides@are dissociated,@which@facilitates@library-based@sequence identification@nd@haracterization@f(PTMs $\Phi 6, @ 1-13]$.

However,@he@fficiency@f@his@pproach@s@educed by@t@east@wo@actors.๑irst,@ompetition@or@rotons@in the@ositive-ion@node@auses@ignal@uppression, @eav-

Address@eprint@equests@o@r.థr.đKjeldsen@andథr.@.@.đensen,థrotein Research@Group,CDepartment@of@Biochemistry@and@Molecular@Biology, University@of@Southern@Denmark,@Campusvej@55,CDK-5230@Odense@M, Denmark.@-mail:®rankk@bmb.sdu.dk,@enseno@bmb.sdu.dk ing@nanyథroteins@ndథost-translational@nodifications either@indetected@r@inreliably@etected@y@nly@ne peptide@ndबimited@mino@cid@equence@nformation. Second,@nanyథPMs@n@oly-peptides@re@abile@s@as phase@cations@and@detach@upon@slight@ion@activation [e.g.,(by@ollision-activated@issociation@(CAD)],@which precludes@dentification@f(\$heir@ite-specific@ocation $\oplus y$ MS/MSథ14].

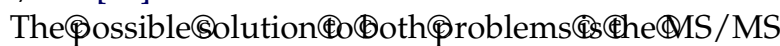
analysis@fegative@ons@s@@omplement@o@MS/MS analysis@of@ations.@With@45\%@of@all@human@protein sequences@s@ell@s@nost@ommonథTMs@eing@cidic, the@xtension@f@nalytical@trategies@o@include@egative peptide@ndథrotein@ons@hould@increase $₫$ he@ensitivity and@amino@acid@sequence@coverage@of@proteins.@In addition, $₫$ heoretical@rypsin@igestion@f@roteins@hows that@t@loes@ot@hift@he@verage@I@alue@f@eptides

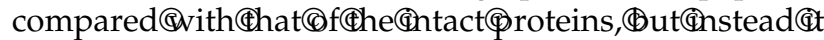
leads@to@a@wider@pI@distribution.@As@a@result@of@the 
specific cleavages of trypsin at the C-terminal side of the basic residues arginine and lysine, basic peptides are preferentially shorter than acidic ones. This size distribution reveals the potential of using acidic peptides for MS/MS as the specificity of protein identification by MS/MS increases significantly with the peptide size [15]. As such, extension of proteome analysis to negative ions should increase both the sensitivity and the validity of protein identification.

Traditional MS/MS techniques utilizing vibrational excitation (VE) of anions often produce insufficient amino acid sequence information. This is due to complex product ion formation from polypeptide anions and it often requires thorough manual interpretation of the tandem mass spectra [16-21]. However, the amino acid sequence information obtained is often found to be complementary to that of CAD in the positive-ion mode [22]. In addition, a recent study demonstrates that CAD of phosphorylated peptide anions reveal information on the site specific location of the phosphorylation when consecutive Thr/Ser amino acid residues are present [23]. Despite the increasing understanding of the fragmentation pattern in CAD of peptides in the negative-ion mode, spectra are complicated by internal ion fragments, abundant side-chain losses and neutral losses from the parent ion $\left(\mathrm{H}_{2} \mathrm{O}, \mathrm{NH}_{3}, \mathrm{H}_{2} \mathrm{O}+\mathrm{NH}_{3}, \mathrm{CO}_{2}+\mathrm{NH}_{3}\right)[24,25]$.

In the negative-ion mode, electron detachment dissociation (EDD) $[26,27]$ has proven to be a potential alternative to CAD for fragmenting peptide poly-anions. EDD utilizes fast $>10 \mathrm{eV}$ electrons to detach electrons from multiple-deprotonated anions. With the formation of electronically excited radical anionic species, spontaneous fragmentation occurs.

$$
\begin{aligned}
& {[\mathrm{M}-\mathrm{nH}]^{\mathrm{n}-}+\mathrm{e}_{\mathrm{hot}}^{-} \rightarrow[\mathrm{M}-\mathrm{nH}]^{(\mathrm{n}-1)^{-\cdot *}}+2 \mathrm{e}^{-}} \\
& \rightarrow \text { peptide fragment ions }(a, x \text { ions })
\end{aligned}
$$

EDD fragmentation is characterized by dominant backbone cleavage of $\mathrm{C}_{\alpha}-\mathrm{C}$ bonds giving $a$. and $x$-type product ions. This allows for efficient peptide sequencing and stands in sharp contrast to the non-specific fragmentation obtained with CAD in the negative ionmode. The formation of predictable product ions in EDD is important as it makes identifications from database searches more reliable. Most importantly, EDD preserves labile PTMs to a great extent. For instance, the site of sulfation was deduced from the EDD spectrum of the sulphated peptide caerulein [26]. However, EDD is a rather inefficient process [28] due to its limited reaction cross section between negatively charged poly-peptides and electrons. As a consequence, accumulation of tens or hundreds of summed mass spectra is often required to obtain satisfactory mass spectral quality. To fully utilize the analytical application of EDD, it is necessary to improve and advance this technology to be compatible with LC-time scale MS/MS experiments. In this paper, we demonstrate a significant increase in the EDD fragmentation efficiency for a large number of peptides. This improvement allows for LCtime scale experiment using short duty-cycle (4-5 s) for MS/MS fragmentation of peptide anions.

\section{Experimental}

\section{Samples and Solutions}

Bovine serum albumin (BSA) was reduced, alkylated, and digested with trypsin (Promega, Madison, WI) using a standard protocol. Phospho-peptides from a trypsin digested protein mixture consisting of 12 proteins [carbonic anhydrase, BSA, $\alpha$-casein (S1 and S2), $\beta$-casein, ovalbumin, $\beta$-lactoglobulin, RNAse $B$, alcohol dehydrogenase, myoglobin, transferrin, lysozyme, $\alpha$-amylase] were enriched using $\mathrm{TiO} 2$ microcolumns [29]. In all experiments, an amount of 700 to $1000 \mathrm{fmol}$ of material was analyzed by LC-EDD-MS/MS.

\section{LC-MS/MS}

A hybrid 7-T linear ion-trap Fourier-transform (LTQFT) mass spectrometer (Thermo Fisher, Bremen, Germany) equipped with an indirectly heated dispenser cathode as an electron source was used for the EDD experiments. Peptides were synthesized in-house or obtained by tryptic digestion of proteins. For off-line experiments peptide solutions were diluted to $\sim 10^{-6} \mathrm{M}$ concentrations. Peptides were injected into the mass spectrometer by ESI using either infusion into a metalcoated nano-ESI-needle (Proxeon, Odense, Denmark) (off-line ESI-MS/MS) or using a nano-flow (250 nl/min) HPLC system (EasyLC, Proxeon, Odense, Denmark) (on-line LC-ESI-MS/MS). Buffer A consisted of $0.1 \%$ formic acid in water. Buffer B consisted of $90 \%$ acetonitrile and $0.1 \%$ formic acid in water. The pre-column and analytical column was packed with $3 \mu \mathrm{m}$ Reprosil C18 AQ (Dr. Maisch, Germany) reverse-phase material. After a $10 \mathrm{~min}$ loading time $(3000 \mathrm{~nL} / \mathrm{min}, 3$ times loading volume) the peptides were eluted from the column with linear gradients over a $45 \mathrm{~min}$ period $(0 \%-45 \% \mathrm{~B}$ in $30 \mathrm{~min}$ and $45 \%-100 \% \mathrm{~B}$ in $4 \mathrm{~min})$ at a flow rate of $250 \mathrm{~nL} / \mathrm{min}$. MS/MS analysis was performed using unattended data-dependent acquisition mode. After a survey scan (300-2000 m/z, 25,000 resolution at $m / z 400$ ) a maximum of two peptides were selected per cycle for EDD-MS/MS (300-2000 m/z, $10,000$ resolution at $\mathrm{m} / \mathrm{z} 400)$. The automated gain control was set to 800,000 ion charges. The maximum accumulation time was set to $800 \mathrm{~ms}$. For each peptide four EDD acquisitions were acquired and summed.

EDD was optimized by varying the irradiation time, electron energy, electron flux, and electron-ion phase correlation. To optimize the electron-ion phase, the delay time for electron injection into the Penning trap was varied. The applied delays were biased by a delay of $43 \mathrm{~ms}$ which was determined by the instrument based on the performance calibration of electron capture dissociation (ECD). EDD fragmentation efficiency was 
determined as the sum of all EDD products ( $(a, x$ ions), except for the charge oxidized species $[\mathrm{M}-\mathrm{nH}]^{(\mathrm{n}-1)-}$, divided by the total sum of all ion signals (eq 1).

$$
\begin{aligned}
& \text { EDD frag } \cdot \text { eff. } \\
& =\frac{\sum \text { EDD products }(a, x \text { and neutral losses })}{\sum \text { total ion abundance }}
\end{aligned}
$$

\section{Results and Discussion}

An increase in EDD fragmentation efficiency was obtained by varying a number of instrumental parameters. The fact that no instrumental modifications were required makes the improvement of EDD straight forward to implement. Earlier studies have shown that EDD is very dependent on both low electron current $(3-10 \mu \mathrm{A})$ and electrons with energy above $10 \mathrm{eV}[26$, 27]. However, in this study we also explore other parameters to increase the EDD efficiency. We optimized EDD by varying the electron irradiation time, electron energy, electron flux, and electron-ion phase correlation and found the following optimum settings: irradiation time (150-170 ms), electron energy of $18 \mathrm{eV}$ and an electron current of 6-8 $\mu \mathrm{A}$, irradiation delay of 40 ms (electron-ion phase correlation). In our experiments, the energy threshold for electron detachment for various peptide anions was determined to 7-9 eV. It should be noted that this energy is substantially higher than the theoretical vertical electron-detachment energy for typical peptide anions (3-4 eV) [27]. However, similar differences of $\sim 7 \mathrm{eV}$ between theoretical and experimentally determined electron-detachment energies has been reported for deprotonated mononucleotides [30].

All experimental parameters were found to be important, however the electron-ion phase correlation was found to be a critical parameter revealing a rather narrow interval for optimal performance. The electronion phase correlation is related to the timing event of electron injection (irradiation) into the Penning trap relative to the spatio-temporal position of the ion cloud. In principle, the overlap between the electron beam used in these experiments and the analyte ion cloud should be complete and along the $\mathrm{z}$-axis of the ICR trap. However, such a perfect alignment is rarely accomplished and causes suboptimal conditions for ion-electron reactions to occur. For instance, ions often deviate from the center of the ICR trap due to induced magnetron motion of the ions, or difficulty of alignment of the dispenser cathode causes the electron beam to be offaxis. A solution to this problem has been the implementation of wider dispenser cathodes [31], which increases the ion-electron overlap. However, despite the observed improvement in the rate of ion-electron reactions, experiments using ECD demonstrated that even wider electron beams cannot compensate fully for the much larger magnetron radius of ions [32]. Therefore, perfect timing translates into maximum overlap between ions and electrons.

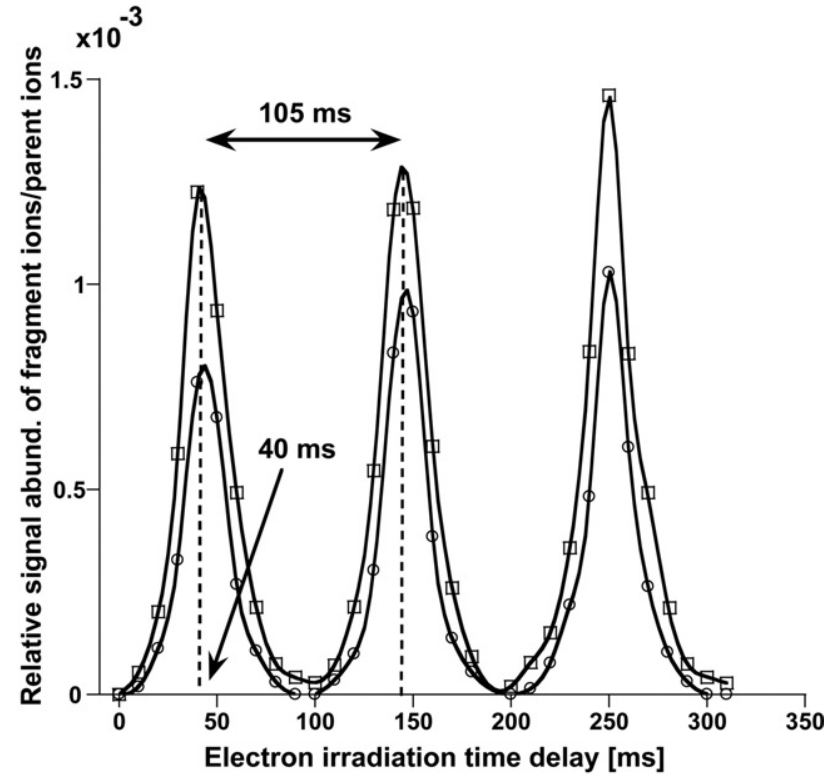

Figure 1. EDD product ion abundances $\left[a_{14}^{-}\right.$(squares) and $x_{13}^{-}$ (circles)] as a function of delay period between ion trapping and electron injection. Data is acquired using $15 \mathrm{~ms}$ irradiation time and delay increments of $10 \mathrm{~ms}$.

To maximize the fragmentation yield in EDD the relative ion signals from two different EDD product ions $\left(x_{13}^{-}\right.$and $\left.a_{14}^{-}\right)$from the phosphopeptide FQpSEEQQQTEDELQDK $(\mathrm{m} / \mathrm{z} 1029, z=2$-) were measured as a function of the delay time for electron irradiation. Figure 1 shows how the abundance of these EDD product ions varies periodically with the electron irradiation delay time. In effect, the product ion abundance increases at least one order in magnitude through a period. The electron irradiation duration was $15 \mathrm{~ms}$, which was the minimum irradiation time that gave measurable product ion signal, and the delay period was varied by increments of $10 \mathrm{~ms}$. The period between maxima was $105 \mathrm{~ms}$ and was found to depend highly on the trapping plate potential (data not shown). Such a periodic dependence is a strong indication that ions in the Penning trap are undergoing magnetron motion. It should be noted that the extent of magnetron motion differs from instrument to instrument since this motion is defined by the trapping voltage, magnetic field strength, distance between trapping plates, and the cell geometry. However, since the magnetron motion is independent of the mass-to-charge ratio of the analyte [33] the same delay for electron injection should be general for all ionic species in the Penning trap. This was confirmed by similar results for a large number of other different mass peptide ions.

In Figure 2 we demonstrate the effect of the optimized electron irradiation delay on the EDD product ion mass spectra of the phosphopeptide FQpSEEQQQTEDELQDK using $0 \mathrm{~ms}$ delay (Figure 2a) and $40 \mathrm{~ms}$ delay (Figure 2b). Both spectra were obtained via static nano-electrospray ion- 


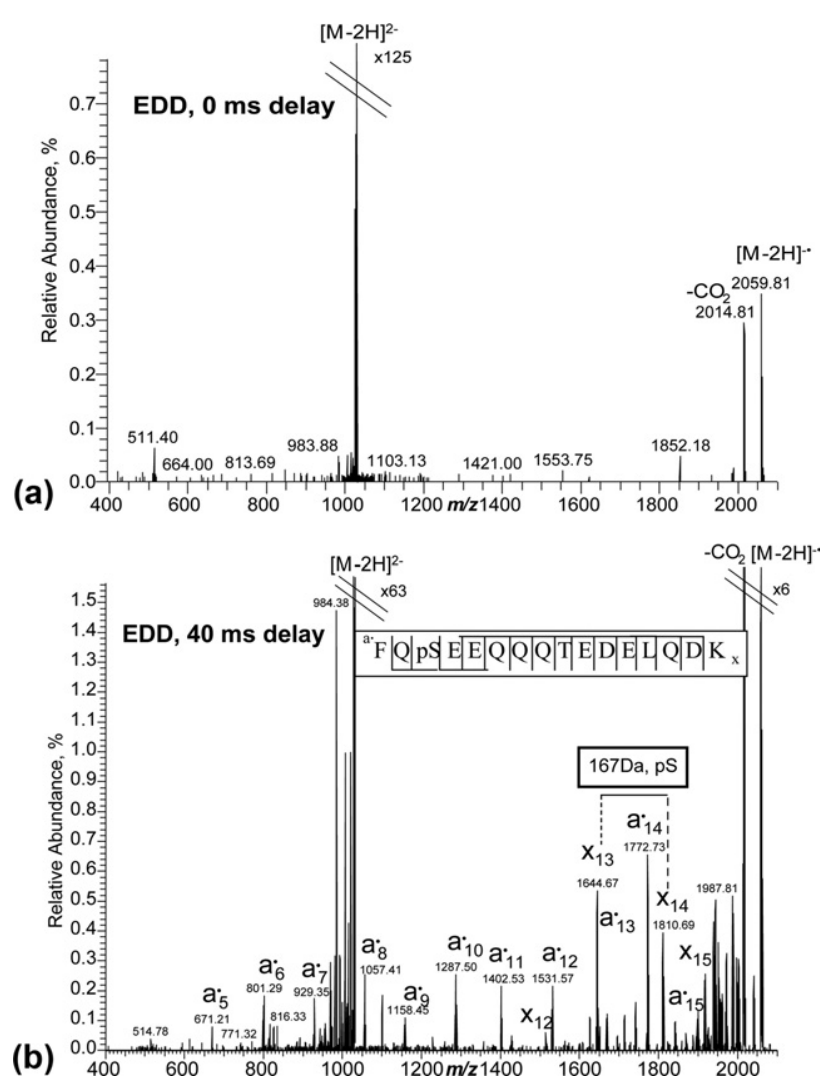

Figure 2. Static nano-ESI-EDD-MS/MS mass spectra of the phosphopeptide FQpSEEQQQTEDELQDK $(m / z$ 1029, $z=2$-) (a) without additional delay and (b) with additional $40 \mathrm{~ms}$ delay. Both spectra contain 100 accumulated acquisitions.

ization and consist of 100 accumulated acquisitions. Using $0 \mathrm{~ms}$ delay, no peptide fragment ions were observed and the signal abundance of the oxidized species $[\mathrm{M}-2 \mathrm{H}]^{-\cdot}$ was less than $1 \%$ of the precursor ions. The abundant loss of $\mathrm{CO}_{2}$ is characteristic for EDD spectra $[26,27]$. Figure $2 b$ represents a delay time point (relative $40 \mathrm{~ms}$ ) where the overlap with analyte ions and the electron beam is maximized. Under these conditions the signal abundance of the oxidized species was $\sim 10 \%$ of that of the precursor ions. Numerous sequence specific product ions were observed $\left(a^{\cdot}{ }_{5}-a^{\cdot}{ }_{15}\right.$ and $x_{12^{-}}$ $x_{15}$ ) covering the complete amino acid sequence of the phosphopeptide and localizing the phosphorylation site to residue $\mathrm{Ser}_{3}$. Using 100 summed acquisitions the fragmentation efficiency was determined to $5.6 \%$. This performance level was obtained even with as few as four summed acquisitions, although fewer than four acquisitions resulted in poor ion statistics and reduced signal-to-noise ratio making assignment of EDD product ions problematic.

This improvement in EDD fragmentation efficiency encouraged us to test the compatibility of EDD with LC-MS/MS. In Figure 3a is shown the base peak chromatogram of tryptic peptides from 1 pmol BSA. Between survey scans peptides of certain signal threshold (10,000 ions) were isolated and subjected to EDD fragmentation. For each peptide four EDD-MS/MS acquisitions were accumulated lasting maximum $5 \mathrm{~s}$ depending on the abundance of the ions (with highabundant ions requiring less accumulation time).

EDD mass spectra of two peptides (HLVDEPQNLIK and VPQVSTPTLVEVSR) are shown as examples in Figure $3 b$ and c. EDD of HLVDEPQNLIK $(m / z 651, z=$ 2-) produced eight inter-residue cleavages out of nine possible ( $N$-terminal cleavage to Pro is immune in EDD) [27]. For the peptide VPQVSTPTLVEVSR $(\mathrm{m} / \mathrm{z} 754, z=$ $2-)$, seven out of eleven possible cleavage products were observed. The fragmentation efficiencies for these two peptides were $\sim 6.5 \%$ and $9 \%$, respectively. Consistent with previous results, even-electron $x$-ions were most frequent and more abundant than oddelectron $a$-ions [27].

A comparison of the results obtained from LCMS/MS experiments using ECD and EDD fragmentation of peptides from 1 pmol tryptic digested BSA is presented in Table 1. The data in Table 1 represent best results from two consecutive injections of the sample using EDD and ECD. EDD sequenced 14 peptides (27\% aa sequence coverage) and ECD sequenced 19 peptides (39\% aa sequence coverage). Seven peptides (18\% aa sequence coverage) were sequenced by both EDD and ECD. Fifty percent of the identified peptides from the EDD experiment were not identified with ECD in the positive-ion mode. This adds additional $19 \%$ of the protein amino acids sequence coverage of BSA, for a total of $58 \%$ amino acid sequence coverage. The relative small overlap demonstrates the complementarity of the ECD and EDD methods and highlights the importance of protein analysis in both positive- and negative-ion mode to approach complete protein characterization, including determination of all PTMs. The fragmentation efficiency of ECD $(15.7 \%$ av.) was superior to EDD (3.6\% av.) and the average peptide amino acid sequence coverage was $78 \%$ with ECD and 50\% with EDD. Based on the base peak chromatogram the signal intensity was on average $10 \%$ to $20 \%$ lower in the negative-ion mode than in the positive-ion mode. This may be explained by the use of acidic mobile-phases that are favorable for reverse-phase LC separation but not optimal for ionization in the negative-ion mode. Attempt was made to circumvent this by applying various basic solutions $(\mathrm{pH}$ 7-8.8), however, the chromatographic performance was significantly affected giving rise to poor peak resolution and thus reduced signal intensity.

Phosphopeptides are generally labile towards loss of phosphoric acid and therefore difficult to sequence with most fragmentation techniques. Phosphorylated peptides have lower $\mathrm{pI}$ than their unmodified analogues, which makes ionization in the negative-ion mode more efficient [34-36]. Thus, fragmentation of phosphorylated peptide ions in the negative-ion mode should be more sensitive than in the positive-ion mode. In Figure $4 \mathrm{a}$ is shown the LC-MS base peak ion chromatogram of a peptide mixture containing $\mathrm{TiO}_{2}$ enriched phosphopeptides $(\sim 1 \mathrm{pmol})$. Phosphorylated peptides were 


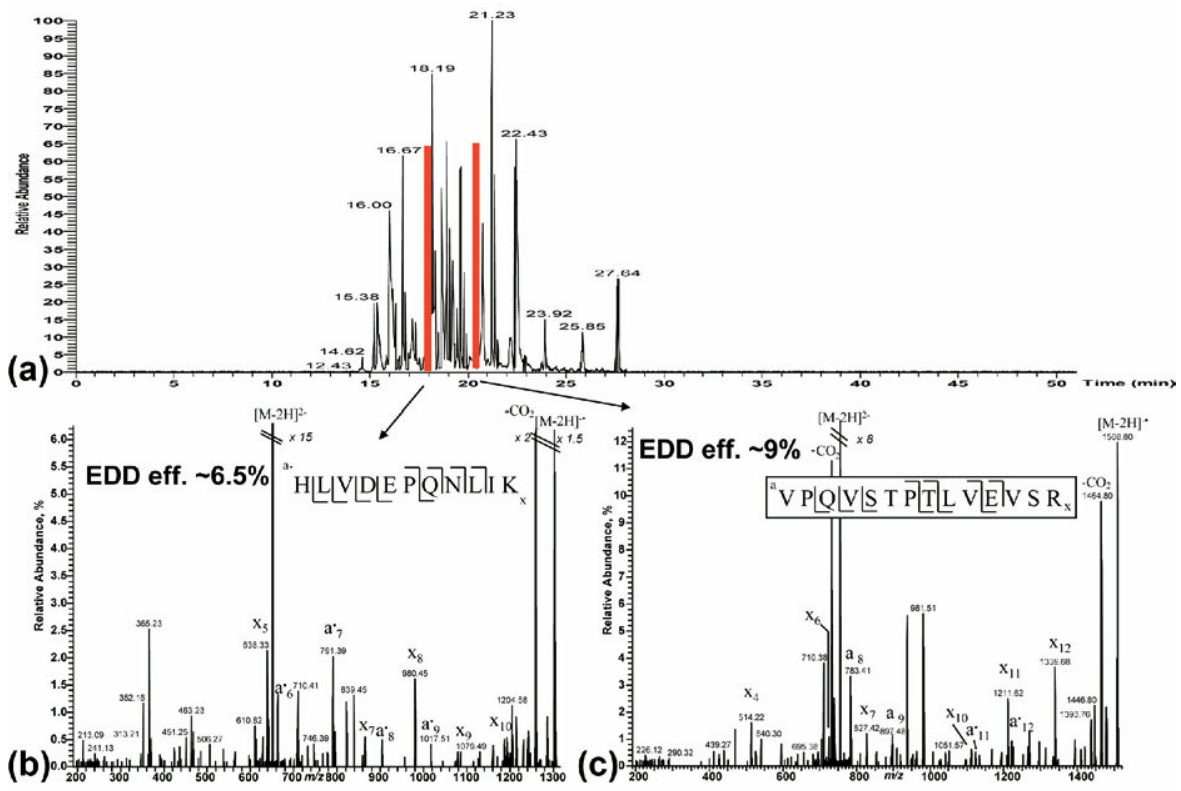

Figure 3. (a) Total ion chromatogram of 1 pmol trypsin digested BSA; (b) EDD mass spectrum of HLVDEPQNLIK $(m / z$ 651, $z=2$-); (c) EDD mass spectrum of VPQVSTPTLVEVSE $(m / z 754, z=2-)$. The cleavage N-terminal to Pro is immune to EDD. All LC-EDD-MS/MS spectra consist of four summed acquisitions lasting maximum $5 \mathrm{~s}$.

enriched from a protein mixture containing 12 proteins of which three are phospho-proteins ( $\alpha$-casein, $\beta$-casein, and ovalbumin). A total of nine phosphopeptides were detected in the negative-ion mode. In Figure $4 \mathrm{~b}$ is shown as an example the LC-EDD-MS/MS spectrum of the doubly phosphorylated peptide EQLPSTpSEENSKK $(\mathrm{m} / \mathrm{z}$

Table 1. Identified peptides from LC-EDD-MS/MS and LC-ECD-MS/MS of 1 pmol tryptic digested BSA

\begin{tabular}{|c|c|c|c|c|c|}
\hline \multirow[b]{2}{*}{ Sequence (BSA, trypsin) } & \multirow[b]{2}{*}{$\mathrm{pl}$} & \multicolumn{2}{|c|}{ EDD } & \multicolumn{2}{|c|}{ ECD } \\
\hline & & $\begin{array}{l}\text { No. cleavages } \\
\text { No. pot. cleavages }\end{array}$ & $\begin{array}{c}\text { Frag. eff. } \\
{[\%]}\end{array}$ & $\begin{array}{l}\text { No. cleavages } \\
\text { No. pot. cleavages }\end{array}$ & $\begin{array}{c}\text { Frag. eff. } \\
{[\%]}\end{array}$ \\
\hline LKECCDKPLLEK & 6.33 & $5 / 11$ & 1.2 & & \\
\hline KVPOVSTPTLVEVSR & 9.75 & $2 / 12$ & 2.1 & & \\
\hline DDPHACYSTVFDK & 4.20 & $3 / 11$ & 1.2 & $7 / 11$ & 10.2 \\
\hline NECFLSHKDDSPDLPK & 4.48 & $4 / 13$ & 0.8 & & \\
\hline ECCHGDLLECADDR & 3.88 & $8 / 13$ & 2.0 & & \\
\hline LVNELTEFAK & 4.31 & $3 / 9$ & 2.8 & & \\
\hline YICDNODTISSK & 3.92 & $6 / 11$ & 4.0 & $8 / 11$ & 15.6 \\
\hline LKPDPNTLCDEFK & 4.35 & $4 / 10$ & 3.9 & $9 / 10$ & 16.7 \\
\hline TCVADESHAGCEK & 4.47 & $7 / 12$ & 4.7 & & \\
\hline EYEATLEECCAK & 3.84 & $6 / 11$ & 3.2 & $8 / 11$ & 10.8 \\
\hline VPOVSTPTLVEVSR & 6.34 & $7 / 12$ & 8.8 & $9 / 12$ & 5.8 \\
\hline LGEYGFONALIVR & 6.93 & $8 / 12$ & 4.6 & & \\
\hline HLVDEPONLIK & 5.25 & $8 / 9$ & 6.2 & $8 / 9$ & 13.4 \\
\hline TVMENFVAFVDK & 4.11 & $7 / 10$ & 5.3 & $8 / 11$ & 12.4 \\
\hline SLHTLFGDELCK & 5.24 & & & $11 / 11$ & 11.9 \\
\hline QEPERNECFLSHK & 5.42 & & & $10 / 11$ & 22.3 \\
\hline ECCHGDLLECADDRADLAK & 4.03 & & & $14 / 18$ & 13.8 \\
\hline DAIPENLPPLTADFAEDK & 3.46 & & & $14 / 14$ & 35.1 \\
\hline RHPEYAVSVLLR & 9.55 & & & $9 / 10$ & 28.1 \\
\hline LCVLHEK & 7.14 & & & $4 / 6$ & 7.3 \\
\hline RPCFSALTPDETYVPK & 6.33 & & & $12 / 12$ & 27.4 \\
\hline KOTALVELLK & 9.57 & & & $6 / 9$ & 23.7 \\
\hline SHCIAEVEK & 5.32 & & & $4 / 8$ & 12.1 \\
\hline CCTKPESER & 6.30 & & & $3 / 7$ & 4.0 \\
\hline YNGVFQECCQAEDK & 3.87 & & & $7 / 13$ & 13.4 \\
\hline EACFAVEGPK & 4.31 & & & $6 / 8$ & 14.9 \\
\hline Average & & & 3.6 & & 15.7 \\
\hline
\end{tabular}




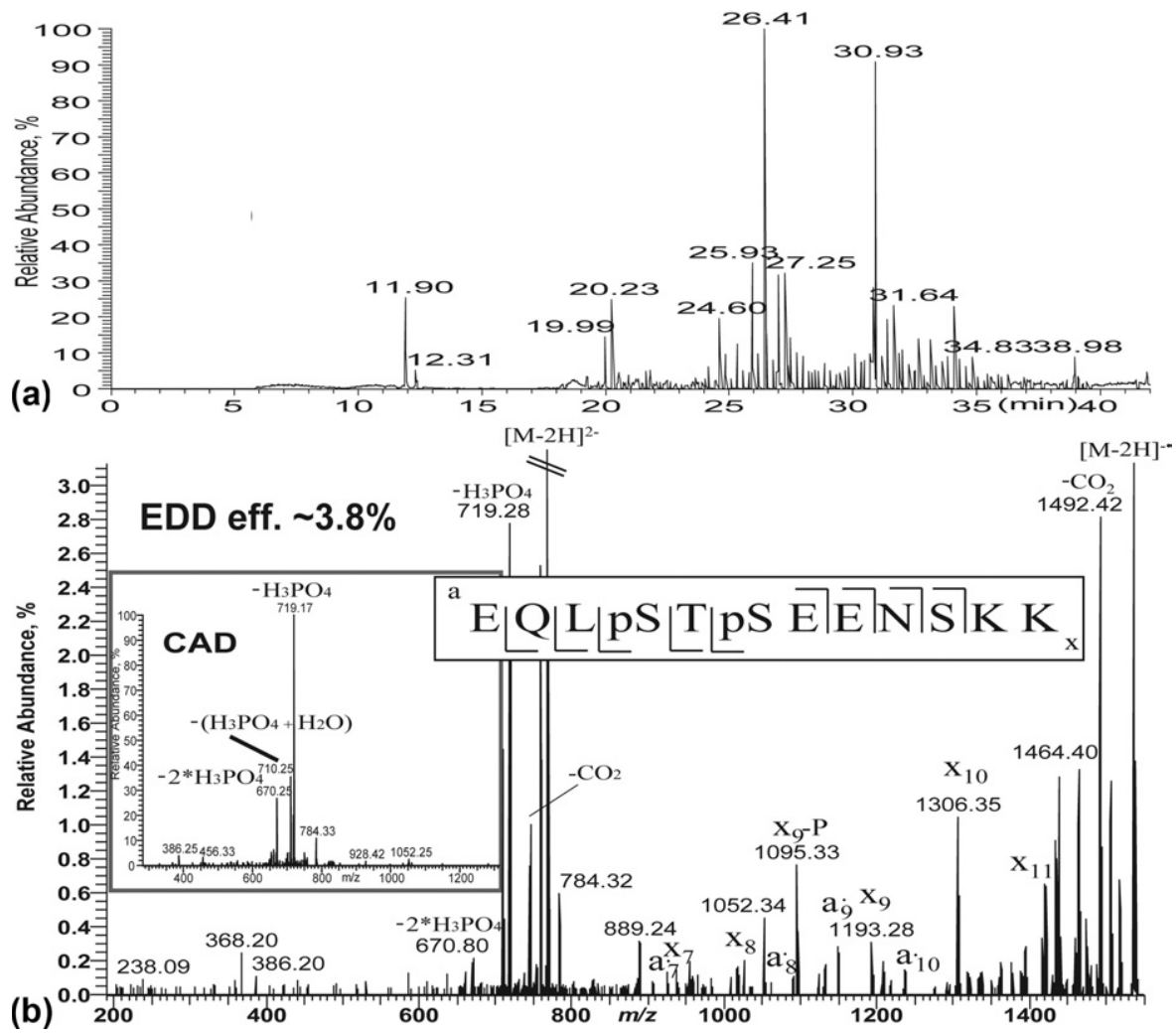

Figure 4. (a) Total ion chromatogram and (b) EDD mass spectrum of the doubly phosphorylated peptide EQLPSTpSEENSKK $(m / z$ 768.3, $z=2$-). All EDD-MS/MS spectra consist of four summed acquisitions.

768.3, $z=2-$ ) containing four potential phosphorylation sites. The extensive EDD fragmentation allowed unambiguous assignment of the two sites of phosphorylation (Ser ${ }_{4}$ and $\mathrm{Ser}_{6}$ ). In comparison, abundant loss of $\mathrm{H}_{3} \mathrm{PO}_{4}$ was observed in negative-ion mode CAD (inset). The loss of $\mathrm{H}_{3} \mathrm{PO}_{4}$ is also present in EDD, but to a much lesser extent (3.5\% of the total ion signal). Nine phosphopeptides were fragmented with EDD and enabled identification and complete characterization of five phospho-peptides. The EDD spectra of the remaining four phospho-peptides contained little sequence information. In comparison, eight phosphopeptides were identified from a similar sample in a recent study from our group using the positive-ion mode and electron-transfer dissociation (ETD) [37]. Although a large overlap (four out of five from the EDD experiment) exists between the identified phospho-peptides in the two studies, the EDD experiment succeeded in identifying one additional phosphopeptide. As with the EDD experiment of BSA, we anticipate that for phospho-peptides the use of basic mobile-phase in LC-EDD-MS/MS would further enhance the signal intensity of these ions and thereby make EDD even more feasible. For instance, Muddiman and Flora have obtained up to a 3-fold increase in the relative ion abundance of phosphopeptides by the addition of 20 $\mathrm{mM}$ piperidine ( $\mathrm{pH}$ 11.8) to the ESI solution [36]. Such a strategy appears to further strengthen the potential of EDD for peptide characterization; however, such strong base is not compatible with most parts of an LC-system and would require an alternative LC-setup or postcolumn addition of alkaline reagents.

\section{Conclusions}

EDD is a gas-phase ion-electron fragmentation technique applicable to multiply charged anions. EDD produces predominantly $a$ - and $x$-ions, which are complementary ions to both ECD/ETD (c- and z-ions) and $\mathrm{CAD}$ (b-and $\mathrm{y}$-ions) produced in the positive-ion mode. Generation of complementary ion pairs increases the confidence of peptide identification [38]. In this study we demonstrated that simple optimization of instrumental parameters is sufficient to increase the fragmentation efficiency of EDD many-fold, making EDD compatible with LC-MS/MS and peptide sequencing at the chromatographic time-scale. The new regime of EDD was tested using peptide- and phosphopeptide mixtures and compared with positive-ion mode sequencing using ECD. As expected, fragmentation using EDD in the negative-ion mode adds complementary sequence information. For BSA, half of the peptides that were identified by the LC-EDD-MS/MS experiment were not identified with LC-ECD-MS/MS in the positive ionmode. Thus, the combination of EDD and ECD increases the overall amino acid sequence coverage of proteins. Phosphopeptide analysis and sequencing in 
the negative-ion mode by LC-EDD-MS/MS was demonstrated, and provided data that augments results obtained by positive ion LC-MS/MS methods using CAD and ETD/ECD. The extension of EDD for on-line time-scale experiments in the negative-ion mode, as demonstrated in this study, holds great promise for a more comprehensive analysis of PTMs and for polypeptide sequencing, and it is a very useful addition to the proteomics research toolbox.

\section{Acknowledgments}

The authors thank Lene Jakobsen for skilled technical assistance. The LTQ-FTICR mass spectrometer was acquired by a generous grant from the Danish National Research Foundation to the Center for Experimental Bioinformatics. This work was supported by the Danish Research Council for Technology and Production Sciences (grant 274-06-0265, F.K.). O.N.J. is a Lundbeck Foundation Research Professor.

\section{References}

1. Lander, E. S.; Linton, L. M.; Birren, B.; Nusbaum, C.; Zody, M. C.; Baldwin; J.; and 259 others; International Human Genome Sequencing Consortium. Initial Sequencing and Analysis of the Human Genome. Nature 2001, 409, 860-921.

2. Jensen, O. N. Modification-Specific Proteomics: Characterization of Post-Translational Modifications by Mass Spectrometry. Curr. Opin. Chem. Biol. 2004, 8, 33-41.

3. Mann, M.; Jensen, O. N. Proteomic Analysis of Post-Translational Modifications. Nat. Biotechnol. 2003, 21, 255-261.

4. Pandey, A.; Mann, M. Proteomics to Study Genes and Genomes. Nature 2000, 405, 837-846.

5. Rajagopal, I.; Ahern, K. Protein Sequencing in the Post-Genomic Era. Science 2001, 294, 2571-2573.

6. Jardetzky, T. S.; Lane, W. S.; Robinson, R. A.; Madden, D. R.; Wiley, D. C. Identification of Self Peptides Bound to Purified Hla-B27. Nature 1991, 353, 326-329.

7. Kelleher, N. L.; Lin, H. Y.; Valaskovic, G. A.; Aaserud, D. J.; Fridriksson, E. K.; McLafferty, F. W. Top Down Versus Bottom Up Protein Characterization by Tandem High-Resolution Mass Spectrometry. J. Am. Chem. Soc. 1999, 121, 806-812.

8. McLafferty, F. W.; Fridriksson, E. K.; Horn, D. M.; Lewis, M. A.; Zubarev, R. A. Biochemistry-Biomolecule Mass Spectrometry. Science $1999,284,1289-1290$.

9. Meng, F. Y.; Cargile, B. J.; Miller, L. M.; Forbes, A. J.; Johnson, J. R.; Kelleher, N. L. Informatics and Multiplexing of Intact Protein Identification in Bacteria and the Archaea. Nat. Biotechnol. 2001, 19, 952-957.

10. Sze, S. K.; Ge, Y.; Oh, H. B.; McLafferty, F. W. Plasma Electron Capture Characterization of Large Dissociation for the Proteins by Top Down Mass Spectrometry. Anal. Chem. 2003, 75, 1599-1603.

11. Mann, M.; Wilm, M. Error Tolerant Identification of Peptides in Sequence Databases by Peptide Sequence Tags. Anal. Chem. 1994, 66, 4390-4399.

12. Aebersold, R.; Mann, M. Mass Spectrometry-Based Proteomics. Nature 2003, 422, 198-207.

13. Boguski, M. S.; McIntosh, M. W. Biomedical Informatics for Proteomics. Nature 2003, 422, 233-237.

14. Monigatti, F.; Hekking, B.; Steen, H. Protein Sulfation Analysis-a Primer. Biochim. Biophys. Acta 2006, 1764, 1904-1913.

15. Cargile, B. J.; Stephenson, J. L. An Alternative to Tandem Mass Spectrometry: Isoelectric Point and Accurate Mass for the Identification of Peptides. Anal. Chem. 2004, 76, 267-275.

16. Steinborner, S. T.; Bowie, J. H. A Comparison of the Positive- and Negative-Ion Mass Spectra of Bioactive Peptides from the Dorsal Secretion of the Australian Red Tree Frog, Litoria rubella. Rapid Commun. Mass Spectrom. 1996, 10, 1243-1247.

17. Steinborner, S. T.; Bowie, J. H. The Negative Ion Mass Spectra of M-H $(-)$ Ions Derived from Caeridin and Dynastin Peptides. Internal Backbone Cleavages Directed Through Asp and Asn Residues. Rapid Commun. Mass Spectrom. 1997, 11, 253-258.
18. O'Hair, R. A. J.; Blanksby, S.; Styles, M.; Bowie, J. H. Characterization of $(\mathrm{M}-\mathrm{H})(-)$ Cations, Radicals, and Anions of Glycine in the Gas Phase: A Combined Experimental and Ab Initio Study. Int. J. Mass Spectrom. 1999, 183, 203-211.

19. Brinkworth, C. S.; Dua, S.; McAnoy, A. M.; Bowie, J. H. Negative Ion Fragmentations of Deprotonated Peptides: Backbone Cleavages Directed Through Both Asp and Glu. Rapid Commun. Mass Spectrom. 2001 15, 1965-1973.

20. Bowie, J. H.; Brinkworth, C. S.; Dua, S. Collision-Induced Fragmentations of the $(\mathrm{M}-\mathrm{H})(-)$ Parent Anions of Underivatized Peptides: An Aid to Structure Determination and Some Unusual Negative Ion Cleavages. Mass Spectrom. Rev. 2002, 21, 87-107.

21. Chass, G. A.; Marai, C. N. J.; Harrison, A. G.; Csizmadia, I. G. Fragmentation Reactions of a(2) Ions Derived from Deprotonated Dipeptides-A Synergy Between Experiment and Theory. J. Phys. Chem. A 2002, 106, 9695-9704.

22. Brinkworth, C. S.; Dua, S.; Bowie, J. H. Backbone Cleavages of [M $\mathrm{H}](-)$ Anions of Peptides. Cyclization of Citropin 1 Peptides Involving Reactions Between the C-Terminal $[\mathrm{CONH}](-)$ Residue and Backbone Amide Carbonyl Groups. A New Type of $\beta$ Cleavage: A Joint Experimental and Theoretical Study. Rapid Commun. Mass Spectrom. 2002, 16, 713-721.

23. Edelson-Averbukh, M.; Pipkorn, R.; Lehmann, W. D. Analysis of Protein Phosphorylation in the Regions of Consecutive Serine/Threonine Residues by Negative Ion Electrospray Collision-Induced Dissociation. Approach to Pinpointing of Phosphorylation Sites. Anal. Chem. 2007, 79, 3476-3486.

24. Bilusich, D.; Brinkworth, C. S.; Bowie, J. H. Negative Ion Mass Spectra of Cys-Containing Peptides. The characteristic Cys $\gamma$ Backbone Cleavage: A Joint Experimental and Theoretical Study. Rapid Commun. Mass Spectrom. 2004, 18, 544-552.

25. Ewing, N. P.; Cassady, C. J. Dissociation of Multiply Charged Negative Ions for Hirudin (54-65), fibrinopeptide B, and insulin A (oxidized). J. Am. Soc. Mass Spectrom. 2001, 12, 105-116.

26. Budnik, B. A.; Haselmann, K. F.; Zubarev, R. A. Electron Detachment Dissociation of Peptide Dianions: An Electron-Hole Recombination Phenomenon. Chem. Phys. Lett. 2001, 342, 299-302.

27. Kjeldsen, F.; Silivra, O. A.; Ivonin, I. A.; Haselmann, K. F.; Gorshkov, M.; Zubarev, R. A. C- $\alpha-C$ Backbone Fragmentation Dominates in Electron Detachment Dissociation of Gas-Phase Polypeptide Polyanions. Chem. Eur. J. 2005, 11, 1803-1812.

28. Kweon, H. K.; Hakansson, K. J. Proteome Res, 2008, 7, 749-755.

29. Larsen, M. R.; Thingholm, T. E.; Jensen, O. N.; Roepstorff, P.; Jørgensen, T. J. D. Highly Selective Enrichment of Phosphorylated Peptides from Peptide Mixtures Using Titanium Dioxide Microcolumns. Mol. Cell. Proteom. 2005, 4, 873-886.

30. Bluhme, H.; Jensen, M. J.; Nielsen, S. B.; Pedersen, U. V.; Seiersen, K. Svendsen, A.; Anderson, L. H. Electron Scattering on Stored Mononucleotide Anions. Phys. Rev. A 2004, 70.

31. Tsybin, Y. O.; Hakansson, P.; Budnik, B. A.; Haselmann, K. F.; Kjeldsen, F.; Gorshkov, M.; Zubarev, R. A. Improved Low-Energy Electron Injection Systems for High Rate Electron Capture Dissociation in Fourier Transform Ion Cyclotron Resonance Mass Spectrometry. Rapid Commun. Mass Spectrom. 2001, 15, 1849-1854.

32. Tsybin, Y. O.; Hendrickson, C. L.; Beu, S. C.; Marshall, A. G. Impact of Ion Magnetron Motion on Electron Capture Dissociation Fourier Transform Ion Cyclotron Resonance Mass Spectrometry. Int. J. Mass Spectrom. 2006, 255, 144-149.

33. Guan, S. H.; Marshall, A. G. Ion Traps for Fourier-Transform IonCyclotron Resonance Mass-Spectrometry-Principles and Design of Geometric and Electric Configurations. Int. J. Mass Spectrom. Ion Processes 1995, 146, 261-296.

34. Froesch, M.; Bindila, L. M.; Baykut, G.; Allen, M.; Peter-Katalinic, J.; Zamfir, A. D. Coupling of Fully Automated Chip Electrospray to Fourier Transform Ion Cyclotron Resonance Mass Spectrometry for High-Performance Glycoscreening and Sequencing. Rapid Commun. Mass Spectrom. 2004, 18, 3084-3092.

35. Bigwarfe, P. M.; Wood, T. D. Effect of Ionization Mode in the Analysis of Proteolytic Protein Digests. Int. J. Mass Spectrom. 2004, 234, 185-202.

36. Flora, J. W.; Muddiman, D. C. Selective, Sensitive, and Rapid Phosphopeptide Identification in Enzymatic Digests Using ESI-FTICR-MS with Infrared Multiphoton Dissociation. Anal. Chem. 2001, 73, 33053311.

37. Kjeldsen, F.; Giessing, M. B. A.; Ingrell, C. R.; Jensen, O. N. Peptide Sequencing and Characterization of Post-Translational Modifications by Enhanced Ion-Charging and Liquid Chromatography Electron-Transfer Dissociation Tandem Mass Spectrometry. Anal. Chem. 2007, 79, 9243 9252.

38. Nielsen, M. L.; Savitski, M. M.; Zubarev, R. A. Improving Protein Identification Using Complementary Fragmentation Techniques in Fourier Transform Mass Spectrometry. Mol. Cell. Proteom. 2005, 4, 835-845. 